\title{
Longo Mai en las luchas socioambientales; perspectivas del colapso de una comunidad rural en Buenos Aires de Puntarenas, Costa Rica
}

\author{
Longo Mai in the socio-environmental struggles; perspectives of collapse in a rural community \\ in Buenos Aires, Puntarenas, Costa Rica
}

\author{
Luis Diego Arias Campos ${ }^{1}$ \\ Recibido: 9/1/2017 / Aprobado: 23/5/2017
}

\author{
Resumen
}

\begin{abstract}
Este trabajo tiene como objetivo describir el caso de la comunidad de Longo Mai como una iniciativa que trata de romper con la dinámica de avance descontrolado de las destrucciones eco sociales que han llevado el planeta al colapso, esto a partir del contexto histórico, observaciones, discusiones, y la metodología relacional de los derechos humanos de Joaquín Herrera Flores. Parte de ese rompimiento se da con la lucha por los derechos humanos, la búsqueda de autonomía, otras formas alternativas al capitalismo y la retroalimentación, ante un contexto en el que pueblos vecinos envueltos en las dinámicas de los monocultivos han mostrado grandes degradaciones sociales, económicas, ambientales, y dependencias de las decisiones de las grandes compañías. El contexto espacial y temporal en el que surge Longo Mai permite percibir las verdaderas dimensiones de dichos colapsos eco sociales, razón por la cual la toma y lucha por la tierra por manos campesinas e indígenas organizadas ha sido y será fundamental para enfrentar las terribles desigualdades y hambrunas en Costa Rica y el mundo.
\end{abstract}

Palabras clave: migración, colapso eco social, Región Brunca, derechos humanos, corporaciones transnacionales.

\begin{abstract}
This work main goal is to describe the case in the community of Longo Mai as an initiative that tries to break with the uncontrolled advance of the ecosocial destructions that have led the planet to collapse; this from the historical context, observations, discussions, and the Joaquín Herrera Flores' relational methodology of human rights. Thus, part of this breakup it is given for the fight of human rights and the quest for autonomy and other alternative ways to capitalism and feedback, in the light of a context in which neighbor villages are involved in dynamics of monoculture that have shown strong social degradations, economic, environmental, and dependency on the decisions of big companies. The spatial and temporal context in which Longo Mai emerges, it allows perceiving the true dimensions of those ecosocial collapses, that is the reason why to take over and to fight for their land by organized peasants and aborigines have been and it will be fundamental to face the terrible inequity and famine in Costa Rica and the world.
\end{abstract}

Keywords: Central American wars; migration; ecosocial collapse; Brunca Region; human rights, ransnational corporations.

\footnotetext{
${ }^{1}$ Coordinador e investigador en el "Programa de Biología Tropical", Escuela de la Tierra, Longo Mai, Costa Rica. Estudiante de Historia, Ciudad Universitaria Rodrigo Facio, Universidad de Costa Rica. Estudiante de Ingeniería en Ciencias Forestales, Campus Omar Dengo, Universidad Nacional, Costa Rica. Correo electrónico : luisdiegoariascampos@gmail.com
} 


\section{Introducción}

Este trabajo surge de la necesidad de alternativas ante el contexto de guerras y despojos que históricamente ha atravesado (para este caso) la región centroamericana y que se han intensificado en los últimos años con la expansión de monocultivos y transnacionales, las guerras por el agua, y las guerras contra el narcotráfico y contra el terrorismo que más bien se materializan en la criminalización de las resistencias sociales antiimperialistas, tomando como base el caso de una comunidad rural en la Zona Sur del país.

El objetivo de este manuscrito es explorar el caso de Longo Mai como punto de resistencia ante las crecientes catástrofes mundiales en el contexto comparativo de la Región Brunca, para comprender la organización comunitaria y la tenencia de tierra como aspectos fundamentales para intentar alcanzar la autonomía y defender los últimos recursos que el hostil capitalismo aún no ha destruido del todo.

Para esto, primero se muestra un marco referencial que abarca el contexto histórico de Longo Mai, así como algunos datos sobre las destrucciones (antrópicas) irreversibles y las luchas hegemónicas que está enfrentando la humanidad y la naturaleza. El siguiente apartado contextualiza las situaciones socioeconómicas que enfrenta la región donde se ubica Longo Mai, para luego abordar la forma en la que, a partir de esas realidades, la comunidad ha buscado alternativas a través de las visiones críticas vivenciadas que parten de un planeta colapsado en el que se vuelve primordial (y a veces imposible) la búsqueda de la dignidad.

\section{Metodología}

Se utiliza la metodología relacional o "impura" de los derechos humanos que propone Joaquín Herrera Flores, ya que plantea establecer los derechos humanos como punto de partida, esto es colocar los "derechos humanos en el espacio donde nos movemos (acción), en la pluralidad (corporalidad) y en el tiempo (historia)". Esto permite plantear métodos multi y transdisciplinarios, ya que las distintas áreas de conocimiento siempre están "contaminadas" del contexto, e hibridizadas entre sí (Herrera-Flores, 2008).

Durante 2016 se realizaron visitas cualitativas mensuales a pueblos vecinos a Longo Mai, específicamente a La Unión, San Pedro, Santa Ana y La Fortuna de San Pedro, para conocer sus dinámicas, organización, dependencia socioeconómica (monocultivos), formas de subsistencia y adaptación a las problemáticas, donde también se realizaron entrevistas a vecinos. Además, se estudió la comunidad de Longo Mai a partir de su contexto histórico, discusiones y observaciones personales en actividades en la comunidad como charlas, conferencias, foros, ferias y otras. Esto permitió analizar de forma comparada la incidencia de la tenencia de tierra, la agricultura de subsistencia, y el trabajo en los monocultivos con la organización comunal, que facilita las luchas de base social ideológicas, agroecológicas y en defensa de los recursos.

Además, se exploraron datos sobre las dimensiones de los colapsos globales, y datos oficiales sobre cultivos de piña y caña, Índice de Desarrollo Humano Cantonal (IDHC), pobreza, desigualdad y desempleo de la Región Brunca, para entender el rezago socioeconómico y degradación ambiental a partir de la agricultura expansiva impulsada desde de los ajustes estructurales que abrieron las puertas al neoliberalismo y a las compañías transnacionales que han monopolizado y explotado los recursos de la zona con más voracidad cada vez.

A manera de contexto espacial, Longo Mai se ubica en Sonador de Buenos Aires de Puntarenas, Costa Rica (coord: $9^{\circ} 15^{\prime} 45^{\prime \prime} \mathrm{N}$ - 8329'32” W). Se encuentra en la región socioeconómica Brunca y región geográfica, el valle de El General. Limita al Este con las plantaciones piñeras de la compañía Pindeco, subsidiaria de Del Monte, límite que en parte es compartido con el río Sonador. Al Oeste limita con el río Convento y con la pequeña comunidad de Cristo Rey. Al sur limita con 
la carretera Interamericana Sur, y al Norte conecta con los bosques protegidos de la cordillera de Talamanca. Cerca de la mitad de este espacio está protegido, lo cual es fundamental en medio de la expansión piñera, y la otra mitad es dedicada a la actividad agropecuaria de subsistencia.

\section{Marco referencial: los orígenes de una comunidad de refugiados}

El movimiento Longo Mai (del provenzal "que dura mucho tiempo"), es originario de Austria, formado por jóvenes de la época del Partido Comunista, que posteriormente formaron Spartakus. Los ataques de la derecha austriaca les motivó a viajar por Suiza, Francia y Alemania, donde otros jóvenes se unieron para compartir ideas (Meijers, 2008). El primer asentamiento de Longo Mai se dio en Francia en 1973, con la iniciativa de "volver a la tierra" mediante un sistema anticapitalista de autosuficiencia agrícola, debido al abandono agrario y la desprotección de los refugiados y obreros (GrafRoben, 2003).

La búsqueda de refugio de nicaragüenses y salvadoreños, relacionado con las guerras o no, motivó al movimiento Longo Mai a adquirir terrenos en Sonador de Buenos Aires de Puntarenas en 1979, que eran dedicados a la ganadería. El objetivo fue establecer un sitio donde lograran tener un estilo de vida autosuficiente. Luego del regreso de los nicaragüenses a su país, después de la guerra, el lugar fue tomado por salvadoreños.

Longo Mai Costa Rica surge debido a que las últimas tres décadas del siglo XX fueron sumamente conflictivas y sangrientas en Centroamérica. La constante incertidumbre por el estallido de la Guerra Fría (Vickers, 2016), la persecución de comunistas y también de cualquier movimiento que no fuese aliado de Estados Unidos, además de las frecuentes insurrecciones, provocaron la muerte y la huida de miles de personas, principalmente a Estados Unidos, México y Costa Rica.
Para el caso de Nicaragua, el golpe contra Anastasio Somoza ocurrió en junio de 1979 con la insurrección del Frente Sandinista de Liberación Nacional (FSLN). La respuesta del gobierno militar con apoyo de la Agencia Central de Inteligencia (CIA), dejó como saldo al menos 50000 personas fallecidas y la huida de muchas otras, sobre todo hacia Costa Rica (principalmente la Región Huetar Norte). Con la toma del poder sandinista hasta 1990 y la reconstrucción del país muchos nicaragüenses refugiados volvieron a su país (Kruijt, 2011).

El caso en El Salvador tiene una realidad similar desde prácticamente la independencia de la Corona Española en 1821. En la década de los 70 se empezó a intensificar la situación con miles de asesinatos por parte del gobierno militar contra los movimientos de resistencia y la población en general. Hacia 1980 junto al asesinato de Monseñor Romero estalló la guerra (no oficial) y la represión de la Fuerza Armada de El Salvador (FAES) contra el insurgente Frente Farabundo Martí para la Liberación Nacional (FMLN), que dejó unos 75000 fallecidos. En el conflicto intervino la Organización de las Naciones Unidas (ONU) con los Acuerdos de Paz de Chapultepec (México) en 1992 (Hayden, 2005).

Costa Rica que funcionaba como base de la CIA, con los movimientos de izquierda reprimidos desde 1948, se mantenía en relativa tranquilidad, a pesar de la crisis del Estado Benefactor. Las luchas por la mejora de servicios y la nacionalización de los privados, fueron las principales razones de las manifestaciones en el país. Esto le permitió a Costa Rica mantenerse al margen de los conflictos armados del resto de Centroamérica y ser parte de los acuerdos de paz durante el primer gobierno de Oscar Arias Sánchez (1986-1990) (Almeida, 2016). Sin embargo, la región continúa siendo sumamente violenta y enfrenta las más terribles injusticias, saqueos y destrucciones, como algunas de las que se verán en el siguiente apartado. 


\section{Capitalismo, despojo y colapso}

Para comprender los orígenes de Longo Mai, partimos del colapso como "la última fase de la vida" de seres vivos, instituciones o cosas (Saxe-Fernández, 2005: 39-40). Es una etapa de catástrofe en la que:

(...) tiende además a reducirse el gradualismo de los procesos, y aumentan "caídas", 'desplomes", derrumbes, extinciones en masa, bombardeos, hambrunas o genocidios en cada vez más países, etc., de carácter repentino, súbito (Saxe-Fernández, 2005: 39-40).

El capitalismo se ha mostrado como la única forma en la que se puede alcanzar la "felicidad", pero con grados de desigualdad y dominio terribles, eventos cada vez más frecuentes e intensos como sequías, inundaciones, plagas, hambrunas, extinciones, etc. (Boff, 1996; Saxe, 2005). Ante el agotamiento, el egoísmo y la violencia aumentan, mientras los recursos se acumulan en cada vez en menos manos (Saxe, 2005), que intentan además extinguir los movimientos sociales que se resisten al saqueo (Global Witness, 2016).

Ante la violencia de las desposesiones y los efectos de los colapsos, los movimientos migratorios son cada vez más frecuentes, donde se violan los derechos a la integridad corporal, a la satisfacción de necesidades, y al reconocimiento (Herrera-Flores, 2008). Estas personas deben enfrentar la xenofobia y los dogmas del miedo a lo desconocido o foráneo, que automáticamente son relacionados con los mitos del peligro, pobreza, delincuencia, terrorismo, etc. (Sandoval et al., 2012).

La premisa del supuesto desarrollo ha convertido al propietario y al campesino en empleado con trabajos esclavizados y mal pagados (desposesión espacial) con plusvalores para los oligopolios, aunque oficialmente las estadísticas sean optimistas. Lo cierto es que -acudiendo a Sojo (2010)-, los datos siempre serán positivos mientras sigan invisibilizándose gran parte de las poblaciones. Esto demuestra que no existe desarrollo sin despojo, exclusión, esclavitud y marginalidad, según Herrera
(2008: 112) “(...) se sigue diciendo, quizá con buena voluntad, que todos 'tienen' los mismos derechos por el simple hecho de haber nacido. ¿Haber nacido dónde?”

De esta forma, lo que anteriormente tomaba una dimensión de crisis, ahora es de colapso, es decir, de no retorno. Tal es el caso de las 768 millones de personas que no tienen acceso a suficiente agua potable segura, según el Fondo de las Naciones Unidas para la Infancia (UNICEF), y 4.8 billones están vulnerables (Vörösmarty et al., 2010), lo que provocó unas 502000 muertes en 2015 (sobre todo niños) (OMS, 2016). En 2015 unas 795 millones de personas fueron afectadas por el hambre; 66 millones son niños (FAO, 2015), mientras que la Organización Mundial de la Salud (OMS) para 2014 cuantificó más de 1900 millones de personas mayores de 18 años con sobrepeso, de las cuales, 600 millones son obesas. Además, 41 millones de niños menores de 5 años tienen sobrepeso u obesidad. Unos 15 millones de niños viven en condición de indigencia, según la Organización de las Naciones Unidas (ONU). En 2015, Estados Unidos vendió 40 mil millones de dólares en armas para sus propias guerras (Global Research, 28-12-2016). Unos 168 millones de menores de edad son esclavos, y cerca de 700 millones de mujeres son casadas forzadamente siendo todavía niñas (TeleSUR, 23-08-2016). En 2015 la pobreza afectó a 175 millones de personas en América Latina (CEPAL, 2016) y según Amnistía Internacional, más de 100 países continúan practicando torturas.

Datos del Banco Mundial muestran que en los primeros ocho meses de 2015 ocurrieron más de 120 desastres relacionados con el colapso climático antropogénico (el concepto "Cambio Climático" es un eufemismo). Además, un aumento de $0.8^{\circ} \mathrm{C}$ por encima de los niveles preindustriales apunta al incremento de catastróficos $2^{\circ} \mathrm{C}$ para mediados de siglo (IPCC, 2014; Banco Mundial, 2014). Los monitoreos de la Administración de la Aeronáutica y del Espacio (NASA) en el Polo Norte revelan la inminente reducción de los casquetes polares. La humanidad genera entre 7000 y 10000 millones de toneladas anuales de basura (Cannon, 2015). Cerca del 80\% de los reservorios de dióxido de carbono $\left(\mathrm{CO}_{2}\right)$ se encuentran en los arrecifes 
de coral, que se están "blanqueando" por la sobrepesca, contaminación, etc. (Hoegh-Guldberg, 1999). Aunque el $\mathrm{CO}_{2}$ es el Gas de Efecto Invernadero (GEI) más abundante, existen otros más contaminantes que no son fijados, pero los inventarios de GEI los interpretan en equivalencia al $\mathrm{CO}_{2}$. Mientras estas situaciones no reciban atención, y los países industrializados no reduzcan emisiones, poca efectividad tendrán iniciativas oportunistas y "ecocapitalistas" como Reducción de Emisiones por Deforestación y Degradación (REDD+) $\mathrm{y}$ algunas otras.

Según Vörösmarty et al. (2010), los ecosistemas dulceacuícolas representan menos del 1\% de la superficie del planeta, pero es donde habita alrededor del $35 \%$ de los vertebrados existentes, de los cuales, entre 10000 y 20000 especies están en proceso de extinción o del todo extintas. Un artículo en Global Research (Burrowes, 12-12-2016), afirma que unas 200 especies de seres vivos desaparecen del planeta diariamente. Las poblaciones animales se han reducido a la mitad en los últimos 43 años (Korenblik et al., 2016; WWF, 2016). Según la Organización de las Naciones Unidas para la Alimentación y la Agricultura (FAO), más de 60 mil millones de animales son producidos anualmente en fábricas para consumo humano, y 167 millones de toneladas de animales son extraídas del agua (FAO, 2016b). En 2015 fueron asesinados 185 activistas, muchos indígenas, campesinos o ambientalistas (Global Witness, 2016).

La presión hegemónica de la acumulación por desposesión definida por Harvey, implica el despojo de los recursos económicos mediante la mercantilizaciónprivatización de todos los productos para subsistir, e incluso de las especies mediante la patentización por parte del Banco Mundial y sus compañías aliadas (Rodríguez, 2012; Delgado, 2015). Es decir, no sólo se es esclavo del trabajo, sino que también del consumo (Boff, 1996; Harvey, 2003).

Intentos más recientes de mercantilizaciónprivatización se han dado con los enjambres de proyectos hidroeléctricos $(\mathrm{PH})$ en todo el mundo como una forma de dominar las poblaciones mediante el acceso al agua. Muchas de estas iniciativas son lideradas por el Banco Mundial, el cual también, junto con el Fondo Nacional de Financiamiento Forestal (Fonafifo), Wealth Accouting and the Valuation of Ecosystems Services (Waves), Banco Central de Costa Rica (BCCR), REDD+ y otras, buscan calcular mediante la Contabilidad del Capital Natural (CCN), el aporte de la biodiversidad sobre la economía (WAVES, 2017). En otras palabras, se trata de economizar y capitalizar la naturaleza para incluirla en el Producto Interno Bruto (PIB) y tomar decisiones a partir de su valor económico, ya sea para la venta, privatización o custodia de quien pueda pagar lo que valga en términos económicos.

Otro espacio despojado ha sido el político; que se ha convertido también en un espacio para la corrupción, vigilancia, militarización, represión y la instauración del miedo como forma de mantener el "orden" (González, 2016). Solo tiene acceso a los puestos políticos quien(es) tengan los medios para pagar campañas multimillonarias, con llamativas vallas publicitarias y perifoneos, o ganar simpatizantes mediante el clientelismo (Acuña, 2009). Según Cerdas (2014), con el quiebre del bipartidismo, hacia tiempos recientes se ha consolidado el Partido Liberación Nacional (PLN) como un único y claro oferente clientelar. Su aparato político posee representación e influencia en espacios locales y regionales, y diferentes niveles jerárquicos. A pesar de eso, la población costarricense tiene el mito fundacional de que, a pesar de los serios problemas y la degradación de lo que se hace llamar democracia, Costa Rica es una "comunidad libre y democrática, pacífica y protectora del medio ambiente" (Estado de la Nación, 2016).

En gran parte de la Zona Sur de Costa Rica, a pesar de dichos mitos, es posible observar las dimensiones de los colapsos mundiales. A pesar de que las luchas antiimperialistas han sido mucho menos sangrientas en Costa Rica, los efectos del neoliberalismo sobre las poblaciones "que no existen" (es decir migrantes, indígenas, campesinos, etc.) sí han sido percibidos notablemente, como se verá a continuación, sobre la Región Brunca. 


\section{Longo Mai bajo el contexto socioeconómico de la Región Brunca}

Longo Mai pretende ser una alternativa a las anteriores situaciones y la excepción de un modelo agrocapitalista que ha marginado a los pequeños productores del Valle de El General y el resto del mundo. La Región Brunca es una de las dos regiones socioeconómicamente más rezagadas del país, la otra es la Región Huetar Norte. Ambas basan sus actividades socioeconómicas en el monocultivo bajo dominio de pocas empresas poderosas. Durante la década de los 70 se impulsó el cultivo de caña en el valle de El General como una forma de diversificación y complemento al café. A finales de esa década, la compañía Pindeco impulsó el cultivo expansivo de piña en Buenos Aires y debido al éxito experimental se expandió a otras zonas.

En pueblos de la Zona Sur se ha fragmentado la organización comunal, se ha implantado la subordinación a los trabajos ajenos y la total dependencia económica de estos, aunque se cuente con espacios para desarrollar actividades propias. Algunos finqueros han tenido que vender sus terrenos principalmente a la compañía piñera, o los han dedicado a los monocultivos, lo que genera riesgo de pérdidas económicas y de cosechas en caso de plagas, sequías, inundaciones, etc. Para evitar estas situaciones, o para aumentar la productividad, se crea dependencia a paquetes tecnológicos agropecuarios con altos costos económicos, sociales y ecológicos, facilitados por el imperio de los agrotóxicos instalado en Costa Rica sin control alguno.

En la producción de la piña se calcula que laboran unas 5852 personas (Humbert y Braßel, 2016), mientras que en la caña los cálculos son más relativos, ya que los trabajos son temporales (enero-abril), pero se calculan (oficialmente) unas 2500 personas (Picado, 20-012016). Muchas son de origen nicaragüense, panameño, salvadoreño o indígena, quienes sufren maltratos laborales y xenofobia. Esto se percibe claramente, por ejemplo, en el trato de (algunos) no indígenas hacia indígenas en la ciudad de Buenos Aires.
De las 37659 hectáreas de piña en el país en 2015, en el Pacífico Sur se cultivan unas 8360 (INEC, 2015b; Humbert y Braßel, 2016). Para 2012, este cultivo representó el 29.7\% de las exportaciones totales en la Región Brunca (MIDEPLAN, 2014). Para 2012 había unas 63315 hectáreas de caña a nivel nacional, de las cuales en Buenos Aires y Pérez Zeledón se encontraban unas 4541 (LAICA, 2013), lo que representó para 2016 una producción de 300 mil toneladas de azúcar, suficiente para hacer circular unos 12 mil millones de colones, y hacer de este cultivo uno de los principales ingresos de la región (Picado, 20-01-2016). A pesar de eso, históricamente se ha dado la proletarización generalizada de la región mediante el enclave productivo (recurrente desde la United Fruit Company), ante la concentración de ingresos en pocas manos y la privación de servicios ambientales por la contaminación o apropiación de recursos (tierra, agua, etc.) (Estado de la Nación, 2008).

Los pocos agricultores que quedan, deben luchar contra los grandes capitales y sus largas cadenas de producción en masa con bajos costos, el poco apoyo y todas las trabas para colocar y certificar sus productos. En la Región Brunca se utilizan alrededor de 13000 hectáreas para únicamente dos cultivos de consumo no primario, es decir, que no son fundamentales para los hábitos alimenticios. Las críticas contra malas prácticas agrícolas como la quema de cañaverales, o acciones de las trans y multinacionales son desmentidas por el gobierno y otros organismos, que ponen por encima la productividad, como ocurrió con el reciente informe de Oxfam sobre la piña en Costa Rica (Humbert y Braßel, 2016; Gutiérrez, 27-10-2016).

De los 81 cantones, son los monocultivistas 2 los que generalmente ocupan los últimos lugares del IDHC. En 2016 la Región Brunca y la Región Huetar Norte, fueron las más desiguales del país. La pobreza alcanzó el 31.2\% de los hogares de la Región Brunca, mientras que la pobreza extrema (ingresos menores al costo de la Canasta Básica Alimentaria (CBA)), afectó al 10.3\% de los hogares de esta región, con una tasa de desempleo abierto de $10.4 \%$, y el menor nivel de ingreso en el país. Estos datos colocan a la Región Brunca -a pesar de 
algunas mejoras con respecto a 2015- como la de mayor pobreza y desempleo del país (INEC, 2015a; INEC, 2016). Además, los bajos índices de escolaridad facilita a las compañías incumplir con los deberes laborales y salariales, debido a la "mano de obra poco calificada", a pesar de las cuantiosas exportaciones (Estado de la Nación, 2012). Es la región en la que menos se cumplen las garantías sociales, y en la que a pesar de contar con la mayor cantidad de fincas del país, los agricultores son el gremio más empobrecido (Estado de la Nación, 2016), además de grandes daños ambientales al aire, al suelo, a los ríos y quebradas, y a las personas por agroquímicos, sedimentación, etc. (Humbert y Braßel, 2016).

Tabla 1. Posición de los cantones de Pérez Zeledón y Buenos Aires en el Índice de Desarrollo Humano Cantonal (IDHC)

\begin{tabular}{ccc}
\hline & IDHC & \\
\hline Año & Pérez Zeledón & Buenos Aires \\
\hline 2010 & 51 & 75 \\
2011 & 53 & 75 \\
\hline 2012 & 52 & 74 \\
\hline 2013 & 58 & 70 \\
2014 & 60 & \\
\hline Fuente: Elaboración propia con datos de UCR y PNUD (2016).
\end{tabular}

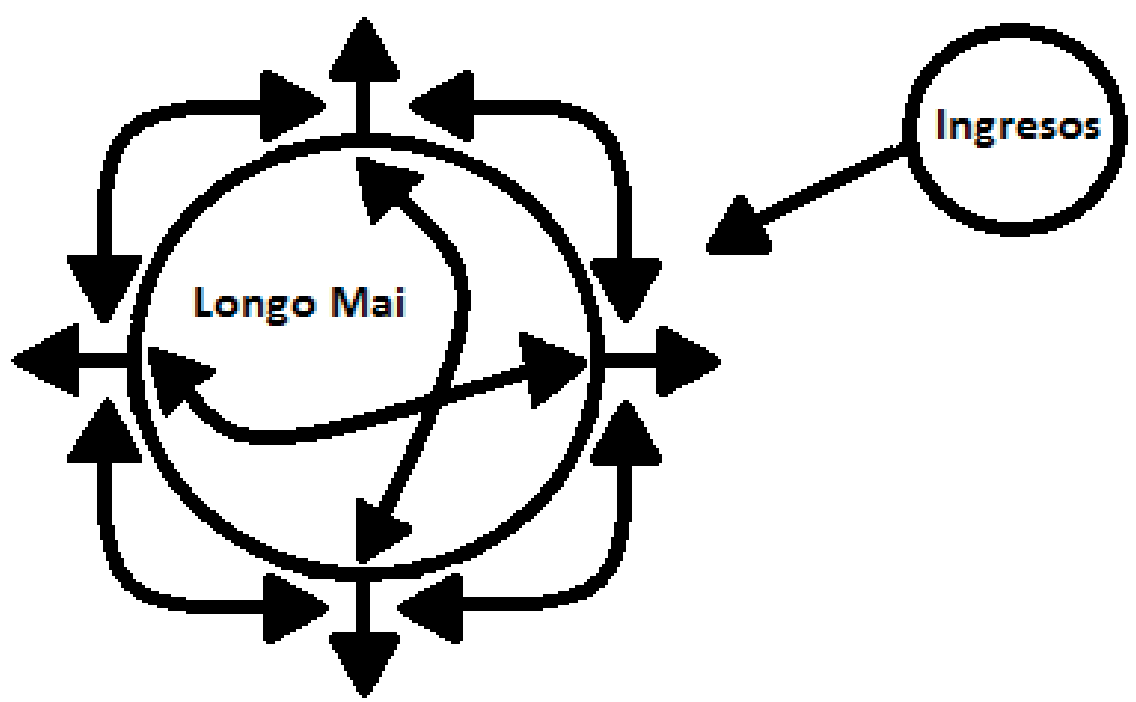

Fuente: Elaboración propia

Figura 1. Percepción de la dinámica económica en la comunidad de Longo Mai, Costa Rica. 


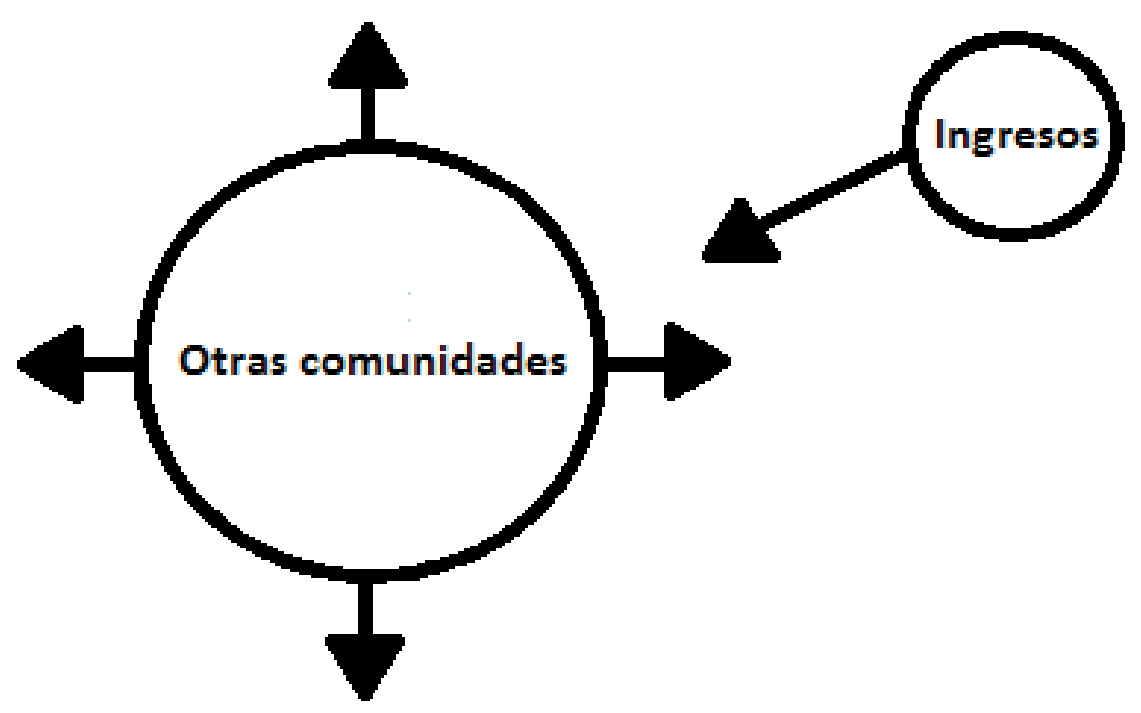

Fuente: Elaboración propia

Figura 2. Percepción de la dinámica económica en otros pueblos del valle de El General, Costa Rica.

Se percibe un flujo de los ingresos mediante la interacción de actividades. Esto se debe a que muchos de los productos, principalmente alimenticios como derivados de la leche, café, carne, hortalizas, frijoles, maíz y otros, es posible adquirirlos en la misma comunidad mediante el trueque, la compra o la autoproducción, ya que recientemente se ha organizado una feria en la que es posible encontrar algunos productos. Esto no solo garantiza un mayor dinamismo de los recursos dentro de la comunidad, sino también productos sanos. Las flechas hacia el exterior indican los bienes no adquiribles en la comunidad.

Al contrario de la dinámica de Longo Mai, los ingresos directamente salen mediante la compra de cualquier producto proporcionado por el libre mercado mundial, siempre con algunas pocas excepciones. Algunos terratenientes se dedican al monocultivo de caña, mas no a productos básicos comestibles, ya que con este libre mercado a veces puede resultar más eficiente comprar que producir, lo que genera el abandono de la tierra. Es decir, los ingresos se fugan casi de inmediato hacia proveedores de bienes y servicios en San Isidro y Buenos Aires, generalmente bajo dominio foráneo, lo cual provoca una economía abierta y dependiente del libre mercado. Un aspecto destacable es la fragmentación de la organización comunitaria desde la decadencia del café y con la llegada de la piña, que ha tenido un resurgimiento con la lucha contra los PH (O. Segura Agüero comunicación personal, 13 de agosto de 2016).

Se ha percibido que esto sucede también por el plustrabajo en las piñeras, ya que las labores y horarios son rotativos y cambiantes, lo que indispone a realizar labores agrícolas propias, ante el poco tiempo libre que es destinado a descansar o al ocio (C. Campos Valverde comunicación personal, 13 de agosto de 2016), que no es más que un breve espacio para recuperarse del agotamiento para volver al trabajo. En ambos casos, un factor común es también el consumo excesivo de alcohol y drogas duras como forma de dispersión, soporte físico y olvido de las intensas y difíciles condiciones laborales y económicas, producto de la influencia cultural construida a partir de los monocultivos (observación personal).

La educación superior (sobre todo privada) ha formado y (a veces) empleado a muchas personas de zonas rurales en el centro de San Isidro. Esta 
educación se percibe como una forma de evitar trabajar la caña y la piña (K. Garro Barrantes comunicación personal, 20 de diciembre de 2016). Queda pendiente estudiar estas dinámicas a fondo.

Estas situaciones adversas representan un reto para las poblaciones/personas que no deseen vender su fuerza de trabajo por un salario que en ocasiones no cubre los gastos médicos de las complicaciones en la salud que implica la exposición al sol y a los agrotóxicos. También representa un reto para quienes poseen necesidades especiales, enfermedades, son adultas o adultas mayores (a veces sin apoyos económicos). Para estos casos, la organización y el "volver a la tierra" se vuelve fundamental.

\section{Organización de Longo Mai}

Para comprender esta comunidad como una alternativa, es necesario conocer su organización. El sistema consiste en arrendamientos de terrenos asignados a familias que pasan por un filtro de estudio socioeconómico y al asignársele la parcela los beneficiarios son libres de realizar cualquier actividad en ella. Si la situación mejora, o no logra adaptarse, cada familia es libre de renunciar.

En 2014, los terrenos boscosos de Longo Mai (452 hectáreas) fueron declarados mediante el Decreto No 38560-MINAE, como Refugio Nacional de Vida Silvestre Privado. El RNVS Longo Mai, adquiere una importancia mayor al ser prácticamente el último remanente en las tierras medias del valle de El General y conectar las zonas bajas con la cordillera de Talamanca. Es visitado por pasantes, voluntarios, turistas y tesiarios principalmente de Austria y Alemania quienes colaboran en los diversos proyectos, de ahí que son comunes las relaciones sentimentales entre europeas y/o europeos con nativos de Longo Mai. Ante esto, existen casos de idealismos sobre Costa Rica, etnocentrismo europeo y otras situaciones de transculturación. Los principales atractivos son los ríos Sonador y Convento, rocas con petroglifos indígenas, la exuberante pluvioselva tropical, recorridos del cacao, café y otros.

\subsection{Turismo rural y agricultura como subsistencia}

Entre las organizaciones comunitarias están la Unión de Amigos para la Protección del Ambiente (UNAPROA), el Comité de Agua, la Junta de Educación, el Patronato Escolar, el Comité Deportivo, el Grupo de Mujeres, la Comisión Defensora de los ríos Convento y Sonador, el Comité de Tu rismo, entre otras. Estas desarrollan actividades en distintas disciplinas en las que los voluntarios, generalmente europeos, colaboran.

La mayoría de beneficiarios utilizan las parcelas para la agricultura de subsistencia, además, Longo Mai ha comenzado a producir su propia marca de café. En esta comunidad el turismo ha sido un incentivo importante de la zona y con esta actividad, administrada por el Comité de Turismo, se benefician muchas de las familias que hospedan visitantes, brindan alimentación (generalmente salvadoreña-costarricense) y demás actividades. En 2004, Longo Mai recibió el premio To-Do Award por el concepto turístico que ha desarrollado.

Partiendo de que unos 500 millones de campesinos no tienen tierra en el mundo, y que el comercio mundial de alimentos está en un grupo muy reducido de empresas (Farah y Vasapollo, 2011), se vuelve necesario expandir, divulgar y motivar técnicas agroecológicas para pequeños espacios y fomentar la economía de solidaridad, cultivando (animales y vegetales) de fácil cuido en comunidades sometidas a las depredaciones de las grandes corporaciones y los gobiernos como facilitadores y cómplices. Esto ha sido una técnica de bajo costo y de fácil manejo que se está implementando en Longo Mai, e incluso en zonas urbanas, y aunque no es una solución absoluta, sí posee múltiples beneficios en cuanto a seguridad alimentaria, tiempo libre, reposo, aprendizaje, amistad, esparcimiento, etc. 


\section{Investigación e iniciativas en la comunidad}

La familia Kolping es uno de los principales enlaces de la comunidad en Europa para motivar la visita a Costa Rica. La población voluntaria europea se compone principalmente de jóvenes que buscan realizar su servicio social, tesis, investigaciones, o voluntariado independiente.

Una de las principales iniciativas es la Escuela de la Tierra; una organización interdisciplinaria fundada como Universidad Alternativa del Sur, durante la visita de François Houtart a los Encuentros con François Houtart: En el Sur las ideas están naciendo, crecen las alternativas, en 2006. La Escuela de la Tierra involucra a personas de la comunidad y visitantes en distintos proyectos relacionados con la protección e interacción con la naturaleza, agroecología, derechos humanos, filosofía, política y otros. Otro proyecto ligado es la Casa Madre Monte coordinado por la folklorista Guadalupe Urbina, quien trabaja en la promoción y desarrollo de habilidades artísticas en personas de la comunidad.

El Circo Fantazztico coordinado por Roland Spendlingwimmer ha involucrado jóvenes de la comunidad y voluntarios latinos en giras internacionales por Europa. En ese sentido, el arte también se vuelve una importante forma de expresión, memoria y sistematización de las luchas sociales.

Es a partir de esta organización y punto de encuentro que ha surgido en gran parte las grandes luchas socioambientales que en los últimos años se han dado en la Zona Sur de Costa Rica. Además de algunos retos a nivel local sobre todo en la protección del RNVS como se verá a continuación.

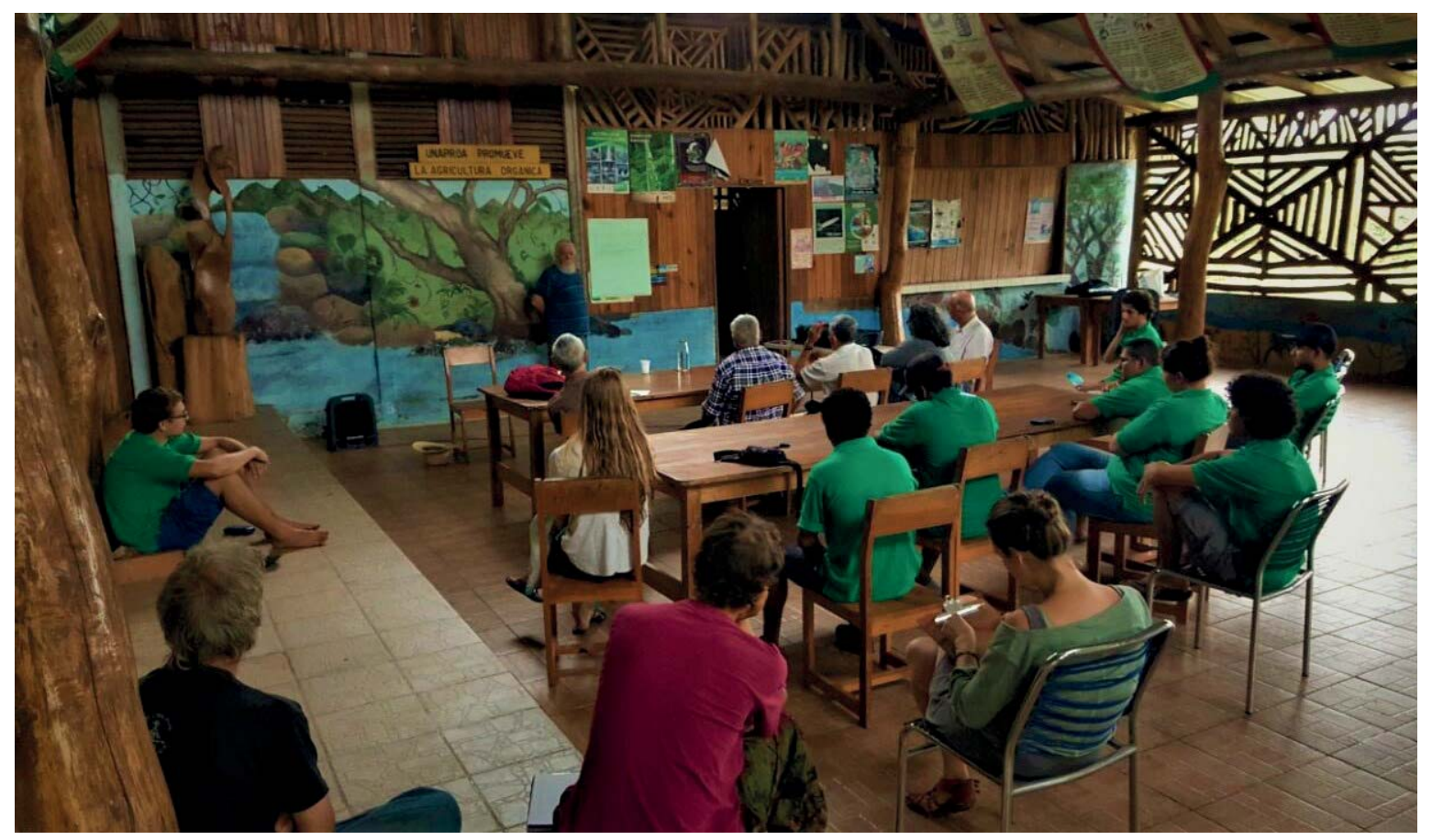

Figura 3. Visita de Scott Pittman de Permaculture Institute a Longo Mai, Costa Rica. Foto de Juan Gómez Meza. 


\subsection{Conflictos y luchas socioambientales}

El RNVS Longo Mai ha experimentado casos de tala ilegal y cacería sobre todo en las zonas más alejadas, donde el Chiricano (Humiriastrum diguense) y Chancho Blanco (Macrohasseltia macrotherantha) son especies extraídas, además de la caza de Tepezcuintles (Cuniculus paca) y otras especies. Se hacen esfuerzos en educación ambiental para reducir estos casos. Otra problemática directa es con las piñeras y la contaminación del río Sonador, ya que constantemente se recorre la cuenca para dar seguimiento de su estado de conservación. El resultado ha sido una evidente degradación principalmente por la sedimentación $\mathrm{y}$ aguas provenientes de las piñeras y de una chilera de varias hectáreas de Del Monte, por los pocos bosques ribereños que son fundamentales para la retención de sedimentos. Las aguas del río constantemente poseen turbiedad y grandes crecidas en época lluviosa por el vertido de aguas pluviales de las piñeras.

En 2012, más de 16 PH fueron propuestos para Pérez Zeledón, Buenos Aires y Coto Brus, dos de ellos (PH Monteverde I y II) para los ríos Sonador y Convento (Spendlingwimmer, 10-042013). Sin embargo, ambos se archivaron en 2015 por la lucha comunal de la Comisión Defensora de los ríos Convento y Sonador, formada por vecinos de Longo Mai y pueblos aledaños, con el apoyo de otras organizaciones como el Movimiento Ríos Vivos. Esta lucha, desde 2002 con el Eco-Foro Socal: ¿¿El agua en manos de quién?" celebrado en Longo Mai, ha sido constante y ha involucrado cada vez más personas a nivel local y nacional (Linder, 2016; Masis-Méndez y Fernández-Vásquez, 2016).

Ya son conocidos los impactos de los $\mathrm{PH}$, sobre todo bajo dominio privado (Anderson et al., 2006; Vörösmarty et al., 2010). La insistencia y la corrupción por seguir con este tipo de energía (a pesar de un superávit de hasta 52.5\% de producción vs. consumo en los últimos años en Costa Rica), sigue generando conflictos y muerte, esto por la relevancia del agua ante el agotamiento del petróleo, además del dominio de la vida de las masas que alcanza quien se apropie de ella, ya que mueve tanto capital, como los contaminantes hidrocarburos (Tablada y Hernández, 2003).

Algunos líderes de estos movimientos también han participado en la lucha contra $\mathrm{PH}$ en otros ríos de la Zona Sur (G. Arias Segura comunicación personal, 21 de junio de 2016), y ha sido ejemplo a nivel nacional de organización y defensa de los recursos (Spendlingwimmer, 1604-2015). La lucha contra los PH ha motivado el seguimiento de las tomas de agua concesionadas por Pindeco en el río Sonador, las cuales se cree que tengan irregularidades en el cumplimiento de los compromisos acordados para aprobar el permiso de extracción de agua.

Esto se suma a los procesos de destrucción percibidos a nivel global, así como las guerras por los últimos recursos y el desencadenamiento de grandes focos de violencia, que como se vio anteriormente, son palpables a nivel local en la Región Brunca, primeramente por el despojo de la tierra ante la implementación del modelo neoliberal, y más recientemente con los intentos de privatización del agua mediante los $\mathrm{PH}$.

\subsection{El colapso como tema central de discusión}

La comunidad recibe grupos de instituciones como la Universidad Nacional de Costa Rica, Universidad de Costa Rica, Universidad Castro Carazo, Permaculture Institute, University of Arizona, University of California State, y otras. Uno de los temas centrales en foros, charlas, conferencias y demás actividades es el colapso mundial, la forma como se han devastado los recursos hasta su extinción y las alternativas existentes para alcanzar la dignidad en un mundo inmerso en la guerra por lo último que queda. 
La perspectiva de los visitantes varía de acuerdo con el contexto de donde provienen y sus influencias ideológicas. Algunos perciben el colapso como un proceso lejano y posible de prevenir, mientras que otros sí lo perciben como la situación actual del planeta. En la visión no tan realista de los colapsos, influye las distracciones capitalistas de voracidad, consumismo y "progreso", además del miedo instaurado por los medios de comunicación como mecanismo de control de las poblaciones para uso como mano de obra y consumo descontrolado.

Un sentimiento frecuente ha sido la percepción de Costa Rica como un país de paz y de igualdad; mitos que deben irse suprimiendo (Sojo, 2010). La mayoría de opiniones sí coinciden en que los espacios como Longo Mai son cada vez más escasos, y que, independientemente, de qué tan cercano perciban el colapso, estas iniciativas de promover el regreso a cultivar la tierra serán fundamentales para la sobrevivencia.

El contexto de la Región Brunca es apto para explicar los antagonismos de la realidad actual. Por un lado, el centro y las periferias de San Isidro con gran desigualdad, familias y barrios de clase media y media-alta, por el otro, familias en condiciones marginadas impactadas por la influencia de las migraciones a Estados Unidos, el coyotaje, el lavado de dinero y el tráfico de órganos. Conforme uno se aleja del centro hacia el sur, empieza a percibir los efectos socioambientales del monocultivo de la piña y la caña en las condiciones de vida de las personas y los grandes deterioros ambientales hasta llegar a Buenos Aires, donde se observa una ciudad notablemente afectada por las piñeras durante décadas.

Algunos miembros de la comunidad son conscientes de que el estado del planeta se encuentra en una fase de no retorno, es decir, de colapso. Los microesfuerzos que puedan realizarse son importantes para que las personas circundantes alcancen la dignidad durante su ciclo de vida, ya que factores políticos y económicos dominan el planeta y a los habitantes, sobre los cuales ejercen poder hegemónico para su opresión, por esta razón, la lucha socioambiental comienza desde el momento de cultivar la tierra, hasta el hecho de evitar someterse al plustrabajo de las grandes compañías, mediante alternativas como la defensa del agua y la naturaleza. Quienes cultiven lo que consumen garantizan una mejor calidad de vida, al ser la agricultura una actividad también filosófica que fomenta la amistad, la solidaridad y la sensibilidad.

\section{Conclusiones}

La organización comunitaria y el acceso a la tierra para la liberación se vuelve fundamental, ya que a pesar de los avances tecnológicos y el 'desarrollo", se sigue esclavizando a millones de personas en el mundo, lo cual muestra que los avances tecnológicos y el capitalismo voraz y monopolizante es la principal causa de la catástrofe ecosocial.

La importancia de Longo Mai a través de la historia puede segmentarse en tres fases del neoliberalismo: 1) Durante la llegada del modelo neoliberal y el refugio a las personas desplazadas. 2) Durante la entrada en operación del neoliberalismo y el rescate de espacios para la agricultura de subsistencia y la conservación, y 3) Durante lo que algunos llaman el posneoliberalismo, la intensificación de los colapsos y la necesidad (global y desesperada) de buscar alternativas.

El abordaje de los colapsos mundiales y la comparación de los antagonismos de la Región Brunca a nivel general y de comunidades rurales, más allá del interés económico, así como desde "los excluidos", permite comprender la forma en la que espacios como Longo Mai pueden representar la excepción a la destrucción, la desigualdad y la esclavitud, que en ocasiones pareciera (y el capitalismo voraz quiere hacer pensar) que es la única alternativa. 
Debido a que las migraciones campociudad han abarrotado los espacios urbanos, y en consecuencia han dejado la tierra en pocas manos hegemónicas, se vuelven necesarios espacios de educación y lucha por los recursos, para la autonomía y la solidaridad agrícola para un "volver a la tierra", más allá de las "ayudas" clientelistas de los gobiernos.

\section{Referencias}

Acuña, I. (2009). "Elementos conceptuales del clientelismo político y sus repercusiones en la democracia”. Rev. Reflexiones 88(2): 27-36.

Almeida, P. (2016)._Neoliberalismo y movimientos populares en Centroamérica. San Salvador: UCA Editores.

Altieri, M. y Toledo, V. (2010). La revolución agroecológica de América Latina: Rescatar la naturaleza, asegurar la soberanía alimentaria y empoderar al campesino". El Otro Derecho 42: 163-202.

Anderson, E., Pringle, C. y Rojas, M. (2006). "Transforming tropical rivers: an environmental perspective on hydropower development in Costa Rica". Aquatic Conserv: Mar. Freshw. Ecosyst. 16: 679-693. DOI: 10.1002/aqc. 806

Banco Mundial. (2014). Bajemos la temperatura. Cómo hacer frente a la nueva realidad climática. Washington: Grupo Banco Mundial.

Boff, L. (1996). Ecología: Grito de la Tierra, grito de los pobres. Buenos Aires: Lumen.

Burrowes, R. (12-12-2016). Life on Earth is Dying. Thousands of Species Cease to Exist. Global Research. [en línea]. Disponible en: https:// goo.gl/lDEs4d
Cannon, T. (ed.). (2015). Global Waste Management Outlook. Viena: UNEP.

Cerdas-Albertazzi, J. (2014). El clientelismo político: Una revisión del concepto y sus usos. Anuario de Estudios Centroamericanos 40: 311-338.

Comisión Económica para América Latina y el Caribe (CEPAL). (2016). Panorama Social de América Latina 2015. México DF: CEPAL.

Delgado, G. (2015). Biodiversidad, desarrollo sustentable y militarización. Esquemas del saqueo en Mesoamérica. México DF: Centro de Investigaciones Interdisciplinarias en Ciencias y Humanidades.

Estado de la Nación. (2008). Decimocuarto Informe Estado de la Nación en Desarrollo Humano Sostenible. San José: Programa Estado de la Nación.

Estado de la Nación. (2012). Decimoctavo Informe Estado de la Nación en Desarrollo Humano Sostenible. San José: Programa Estado de la Nación.

Estado de la Nación. (2016). Vigésimo Segundo Informe Estado de la Nación en Desarrollo Humano Sostenible. San José: Programa Estado de la Nación.

Farah, I. y Vasapollo, L. (coords.). (2011). Vivir Bien. ¿Paradigma no capitalista? La Paz: CIDESUMSA.

Global Research. (28-12-2016). US Sold \$40 Billion in Weapons in 2015, Tops Global Arms Market. Global Research. [En línea]. Disponible en: https://goo.gl/NV3vCc

Global Witness. (2016). En terreno peligroso. London: Global Witness.

González-Hernández, B. (2016). “Mesoamérica como espacio mortal: Dependencia estratégica y biogeopolítica del caos". Intellector 13(25): 115-129. 
Graf-Roben, M. (2003). "Evropska Kooperativa Longo Mai: Ideje I Praksa”. Rec 72(18): 337405.

Grupo Intergubernamental de Expertos sobre Cambio Climático (IPCC). (2014). Cambio climático 2014: Impactos, adaptación y vulnerabilidad. Ginebra: IPCC-OMMPNUMA.

Gutiérrez, T. (27-10-2016). “Gobierno contradice informe de Oxfam: 'piña cumple estándares de calidad"'. Crhoy.com. [en línea]. Disponible en: https://goo.gl/pp4r8T

Harvey, D. (2003). The New Imperialism. New York: Oxford University Press.

Hayden, B. (2005). Salvadoreños en Costa Rica. Vidas desplazadas. San José: Editorial UCR.

Herrera, J. (2008). "La complejidad de los Derechos Humanos. Bases teóricas para una redefinición contextualizada”. Revista Internacional de Direito e Cidadania 1: 103-135.

Hoegh-Guldberg, O. (1999). "Chimatic change, coral bleaching and the future of the world's coral reefs". Mar. Freshwater. Res. 50: 839-866.

Humbert, F. y Braßel, F. (2016). Süße Früchte, bittere Wahrheit. Die Mitverantwortung deutscher Supermärkte für menschenunwürdige Zustände in der Ananas- und Bananen produktion in Costa Rica und Ecuador. Oxford: Oxfam Deutschland.

Instituto Nacional de Estadística y Censo (INEC). (2015a). Encuesta Nacional de Hogares 2015. San José: INEC.

Instituto Nacional de Estadistica y Censo (INEC). (2015b). VI Censo Nacional Agropecuario. San José: INEC.

Instituto Nacional de Estadística y Censo (INEC). (2016). Encuesta Nacional de Hogares 2015. San José: INEC.
Korenblik, A., Leggett, T. y Shadbold, T. (2016). World Wildlife Crime Report 2016: Trafficking in Protected Species. Viena: United Nations Office on Drugs and Crime.

Kruijt, D. (2011). “Revolución y contrarrevolución: el gobierno sandinista y la guerra de la Contra en Nicaragua, 1980-1990". Desafíos 23(2): 5381.

Liga Agrícola Industrial de la Caña de Azúcar (LAICA). (2013). ¿Cómo se distribuye y donde se cultiva territorialmente la caña destinada a la fabricación de azúcar en Costa Rica? [En línea]. Disponible en: https://goo.gl/QGSK40

Linder, R. (2016). Die Auswirkungen der Privatisierung der Stromerzeugung auf das Menschenrecht Wasser - das Beispiel Longo Mai, Costa Rica. Masterbeit. Universitat Passau, Passau.

Masis, M.J. y Fernández, D. (coords.) (2016). Defensa de los ríos Convento y Sonador. La historia de nuestra lucha. San José: Vicerrectoría de Acción Social-UCR.

Meijers, C. (2008). "Longo maï, un projet écologique?” Réfractions 18: 23-40.

Ministerio de Planificación Nacional y Política Económica (MIDEPLAN). (2014). Región Brunca. Plan de Desarrollo 2030. San José: MIDEPLAN.

Organización de las Naciones Unidas para la Alimentación y la Agricultura (FAO). (2015). Estado de la inseguridad alimentaria en el mundo 2015. Roma: FAO.

Organización de las Naciones Unidas para la Alimentación y la Agricultura (FAO). (2016a). Cambio climático y seguridad alimentaria y nutricional América Latina y el Caribe (orientaciones de política). Santiago: FAO. 
Organización de las Naciones Unidas para la Alimentación y la Agricultura (FAO). (2016b). El estado mundial de la pesca y la acuicultura 2016. Contribución a la seguridad alimentaria y la nutrición para todos. Roma: FAO.

Organización Mundial de la Salud (OMS). (2016). Agua: Datos y cifras. OMS. [En línea]. Disponible en: https://goo.gl/ftzACH

Rodríguez, S. (2012). El despojo de la riqueza biológica: de patrimonio de la humanidad a recurso bajo soberanía del Estado. México DF: Editorial Îtaca.

Picado, C. (20-01-2016). Unos 2500 empleos genera la zafra en Pérez Zeledón. TVSur. [En línea]. Disponible en: https://goo.gl/u2smtE

Sandoval, C., Brenes, M. y Paniagua, L. (2012). La dignidad vale mucho. San José: Editorial UCR.

Saxe, E. (2005). Colapso mundial y guerra. San José: Editorial Amo al Sur.

Sojo, C. (2010). Igualiticos: la construcción social de la desigualdad en Costa Rica. San José: PNUD.

Solano, J. y Villalobos, R. (s. f.). Regiones y subregiones climáticas de Costa Rica. San José: Instituto Meteorológico Nacional.

Spendlingwimmer, J. (10-04-2013). "Efectos de los proyectos hidroeléctricos Monteverde". La Nación. [En línea]. Disponible en: https:// goo.gl/UcG4Gm

Spendlingwimmer, J. (16-04-2015). "Ríos secos o casi secos: el caso del río Convento en la Zona Sur”. Diario Extra. [En línea]. Disponible en: https://goo.gl/9DtPUZ

Tablada, C. y Hernández, G. (2003). Petróleo, poder y civilización. La Habana: Editorial de Ciencias Sociales.
TeleSUR (23-08-2016). "Hay casi 49 millones de esclavos en el mundo en pleno siglo XXI". TeleSUR. [en línea]. Disponible en: https:// goo.gl/1wWnu7

Universidad de Costa Rica (UCR) y Programa de las Naciones Unidas para el Desarrollo (PNUD). (2016). Atlas de Desarrollo Humano Cantonal. [En línea]. Disponible en: https://goo.gl/ o3DcE2

Vickers, R. (2016). "Intelligence and Punta Huete Airfield: A Symbol of Past Soviet/Russian Strategic Interest in Central America". Studies in Intelligence 60(2): 13-24.

Vörösmarty, C., McIntyre, P., Gessner, M., Dudgeon, D., Prusevich, A., Green, P., Glidden, S., Bunn, S., Sullivan, C., Liermann, C. y Davies, P. (2010). "Global threats to human water security and river biodiversity". Nature 467(7315): 555-561.

Wealth Accounting and the Valuation of Ecosystem Services (WAVES). (2017). WAVES y la contabilidad de la riqueza. [En línea]. Disponible en: https://goo.gl/wZDhhn

World Wide Foundation (WWF). (2016). Living Planet Report 2016. Risk and Resilience in a New Era. Gland: WWF International. 